\title{
SYMPOSIUM I. Genetic Heterogeneity
}

SI-1. MOLECULAR HETEROGENEITY OF $\beta$-THALASSEMIA. Yasuyuki FUKUMAKI (Res. Lab. Genet. Inf., Kyushu Univ., Fukuoka 812)

$\beta$-Thalassemia is a heterogenous group of hereditary diseases characterized by reduced or absent production of $\beta$-globin chains. The disease has a high frequency in regions endemic for malaria including the Mediterranean Basin, Africa, middle East, Southeast Asia and South China. To understand molecular heterogeneity and to set up a prenatal diagnosis program, we have done a systematic survey of the $\beta$-thalassemia mutations among patients in Asian countries using PCR-related techniques. We found eleven different mutations in Thailand, eleven different ones in Malaysia, three in Taiwan and nine in Japan. We also analyzed chromosome haplotypes designated by RFLPs within the $\beta$ globin gene cluster bearing each mutation. Origins and migration of mutations in Asian populations were presented. Three different 3rd exon mutations detected in Japanese $\beta$-thalassemia patients were discussed in terms of malaria hypothesis.

\section{SI-2. GENETICS AND PATHOLOGICAL PROCESS OF FAMILIAL AMYLOIDOTIC} POLYNEUROPATHY. Shuichiro MAEDA and Ken-ichi YAMAMURA (Kumamoto Univ. Med. Sch., Kumamoto 862)

Amyloidosis comprises heterogenous group of disorders characterized by extracellular or intracellular deposition of amyloid fibrils. Familial amyloidotic polyneuropathy (FAP) is one type of systemic amyloidosis showing an autosomal dominant inheritance and is characterized by prominent autonomic and peripheral neuropathy. FAP is classified into four groups (type I-IV) depending on clinical symptoms. Recent biochemical and molecular analyses have revealed that two types (type I, II) were caused by the mutation of transthyretin (TTR) gene, type III by the mutation of apolipoprotein gene, and type IV by the mutation of gelsolin gene. By the use of cloned TTR gene it is now possible to carry out DNA diagnosis for FAP. The result obtained by DNA diagnosis showed that there is a big variation of the age at onset suggesting that other factor is involved in the development of FAP. Experiments on FAP transgenic mice demonstrated that environmental factor is involved in the onset of amyloid deposition.

\section{SI-3. GENETIC HETEROGENEITY AND MOLECULAR PATHOLOGY OF FABRY} DISEASE. Hitoshi SAKURABA (Dept. Clin. Genet., Tokyo Metr. Inst. Med. Sci., Tokyo 113)

Fabry disease is an X-linked recessive lysosomal storage disorder caused by the deficiency of $\alpha$-galactosidase A ( $\alpha-\mathrm{Gal}$ A). Classically affected males have no detectable $\alpha$-Gal A activity and developed clinical manifestations such as pain, angiokeratoma, hypohidrosis, corneal clouding and vascular diseases of the central nervous system. Recently a variant group of patients who presented only with myocardiac manifestations after 50 years of age and with a significant residual enzyme activity were found. Molecular study revealed that nonsense mutation, splicing mutation and gene rearrangements 
including deletion/insertion caused classical form Fabry disease and an amino acid substitution in exon 6 resulted in cardiac form Fabry disease. Fabry disease comprises heterogeneous mutations at the molecular level.

\section{SI-4. GENETIC HETEROGENEITY IN HM1 GANGLIOSIDOSIS-HETEROGE-} NEITY IN THE ENZYME PROTEIN LEVEL. Tatsuro MUTOH and Akira TOKUDA (2nd Dept. Intn. Med., Fukui Med. Sch., Fukui 910-11)

Genetic lack of acid $\beta$-galactosidase causes a wide variety of clinical phenotypes such as three forms of GM1 gangliosidoses and Morquio B syndrome. The reduction of the activity of this enzyme results in the accumulation of glycoconjugates such as GM1 ganglioside and glycoprotein having $\beta$-galactose at the non-reducing and in the brain and systemic organs. All forms of GM1 gangliosidosis show moderate to severe neurological problems, but the severity of physical symptoms differs in each subtypes. On the other hand, Morquio B syndrome exhibits physical manifestations without any CNS involvements. At present, the biochemical and molecular basis underlying these different clinical phenotypes remains unknown. We have studied the enzyme protein present in the skin fibroblasts from the patients with this disorder biochemically and enzymatically. These studies have revealed that patients' enzyme from these subtypes have altered properties from those of controls and also differs from each other (i.e. substrate specificity, altered Michaelis constant, optimum $\mathrm{pH}$, and heat stability). We also examined the nature of patient's enzyme using purified preparations. The data clearly showed that patient's enzyme is mutant enzyme. These studies indicate that there exists heterogeneity in the enzyme protein level.

Our subsequent neuropathological and biochemical studies in conjunction with other reports on the adult form of this disorder raised another important question that what is the biochemical and molecular basis for the selective accumulation of GM1 ganglioside in the specific regions of the patients' brain, although severe reduction of the enzyme activity is evident in all regions of the brain. At this symposium, we discussed the possible cause for this selectivity in neuropathological involvements.

\section{SI-5. PHENOTYPE-KARYOTYFE CORRELATIONS AMONG CHROMOSOME} ANOMALIES AND ITS HETEROGENEITY. Hidetsune OISHI (Dept. Genet., Inst. Develop. Res., Aichi Pref. Colony, Kasugai 480-03)

Chromosome abnormalities found in congenital anomalies result from unbalanced changes of chromosome number or structure. These chromosomes in the unbalanced condition were sporadically produced in parental germ cells, or inherited one of the abnormalities from a parent with balanced condition of chromosome rearrangements, such as reciprocal translocation, inversion or insertion. The each excess or lack chromosome of the patients obviously contained the common genes. In addition, new mutations on the intact gene may occur at the points of chromosome breaks prior to the structural rearrangement. Activity of these genes, including newly formed ones, reflects to phenotype formations. Various clinical features are observed in patients with aberrations of chromosome number and without any chromosomal breakage. Therefore it is considered 
that the process of normal phenotype formations are disturbed under the genetic imbalance by excess or lack of many intact genes, though most of these genes might keep normal functions.

Apparently similar features are observed in various clinical aspects of patients with a certain type of the chromosome abnormality, but not in all cases. On the other hand, some similar features are also found in patients with various chromosome abnormalities and with different types of syndrome. It is very difficult to clarify the cause of every clinical features of the patients since these similar phenotypes by different genes are indistinguishable from each other.

In general, it is expected to know the genotype by chromosome examinations of malformed children, while their phenotypes are supposed on these clinical features. However, it is unable to detect any minor changes of the chromosomes in the gene size and some differences of clinical aspects are observed in the same chromosome abnormalities. In these cases, the chromosome configuration also indicates simply a kind of phenotypes. As a familiar instance, it is noticed normal to abnormal individuals with balanced autosomes of reciprocal translocations. Even if the clinical features are normal, their fertilities are different in its degree. By breakpoint analyses of the structural rearrangement along chromosome length in 3 groups of recurrent abortion (RA), only a parent with balanced condition (OB) and balanced carriers for two or more generations (BB), it is suggested that some chromosome arms are preferentially involved and that the breakpoints are not distributed at random on the chromosome arms. These tendencies in cases of $\mathrm{OB}$ and $\mathrm{BB}$ are much higher than that in RA, and characteristic excess or dearth of those is also noticed on same 11 arms of groups $O B$ and $B B$. From these data it is concluded that the most cases of $\mathrm{OB}$ and $\mathrm{BB}$ are classified in the same category and these of RA in the other. In these families, the proportion of mother is 2 times larger than that of father in these balanced carriers ( $\mathrm{OB}$ and $\mathrm{BB}$ ) ascertained through probands.

These data of similarity on clinical features and preservation of abnormal chromosomes in these families are very useful to study more in detail, though these are still obscure in regard to the heterogeneity.

SI-6. GENETIC HETEROGENEITY IN RETINITIS PIGMENTOSA. Keiko FUJIKI and Yoshihiro HOTTA (Dept. Ophthalmol., Juntendo Univ., Tokyo 113)

Retinitis pigmentosa is a group of clinically and genetically heterogeneous disorders of the retina, characterized by initially night blindness, progressive visual field loss, midperipheral intraretinal bonespicule pigmentation, attenuation of retinal arterioles, and an abnormal or unrecordable electroretinogram (ERG).

Typical retinitis pigmentosa (RP) unassociated with other abnormalities is also genetically heterogeneous. The prevalence of RP has been calculated to be worldwide 1 in 4000 to 1 in 7000 persons. The proportion of the different genetic types of RP was calculated to be approximately 50\% autosomal recessive (ARRP), 10-20\% autosomal dominant (ADRP), 5\% X-linked (XLRP), and 30-40\% sporadic or isolated cases by inbreeding and segregation analysis of 385 probands from our clinic during the last 9 years.

Genetic heterogeneity exists also within each genetic type. Multilocus homogeneity test from linkage studies of 62 family pedigrees with XLRP by Ott et al. (1990), revealed 
the presence of two XLRP loci and gave estimates for their map location. The location of XLRP gene was estimated at $1 \mathrm{cM}$ distal to locus OTC in $60-70 \%$ of the families with XLRP, and halfway between DXS14 (p58-1) and DXZ1 (Xcen) in 25-40\% of the families with XLRP. An estimated recombination fraction between the two XLRP loci was $25 \%$, and the third locus in midway between DXS28 (C) and DXS164 (pERT87) was also supported. No correlation was detected between the clinical manifestations of XLRP and the two different loci (Xp21 and Xp11) (Chen et al., 1989).

A large pedigree of Irish origin with early onset ADRP has been found to be closely linked to D3S43(C17) from the long arm of chromosome 3 (McWilliam et al., 1989), while C17 was unlinked in a late onset pedigree (Inglehearn et al., 1990). Dryja et al. (1990) found out a mutation of rhodopsin gene which is located at the long arm of chromosome 3 in ADRP patients. The alteration was a base transversion of $\mathrm{C}$ to $\mathrm{A}$, resulting in the change of a proline to a histidine residue at position 23 in $17(11.5 \%)$ of 148 unrelated patients with ADRP and not in any of 102 unrelated normal individuals. They suggested another three point mutations in the rhodopsin gene of patients with ADRP in ARVO meeting at Sarasota, 1990. We tried to find out the mutation in Japanese patients with ADRP and found a family (mother and her son) with the mutation in position 347 among 13 unrelated families including 17 patients with ADRP. Although the mechanism by which this mutation leads to widespread degeneration of both rod and cone photoreceptors is still unknown, these findings should be the first step to approach the pathogenesis of RP.

Gyrate atropy (GA) is one of the related disorders of retinitis pigmentosa, and a rare and an autosomal recessive chorioretinal degenerative disease caused by deficiency of the mitochondrial enzyme, ornithine aminotransferase (OAT). A cDNA for human OAT has been cloned and characterized (Inana et al., 1986) and the functional OAT gene sequences have been assigned to 10q26 (Barrett et al., 1987).

A patient with both a partial heterozygous deletion of functional OAT gene and a complete absence of the OAT mRNA was identified (Inana et al., 1988). Results of Southern blot, Northern blot and enzyme assay of the patient, his father and mother, two sons and a daughter clearly demonstrated the presence of two distinct heterozygous defect in OAT gene in the parents of the patient, their coming together to result on the loss of both functional alleles in the patient, and their transmission to the children to establish the separate heterozygous states (Hotta et al., 1989).

Using RNase A protection assay of GA patient fibroblast RNA and/or PCR and genomic sequencing, at least 16 point mutations of the functional OAT gene have been detected (Ramesh et al., 1988; Mitchell et al., 1989a and 1989b; and Inana et al., 1989). Among them, point mutations of codon 271 (arginine $\rightarrow$ lysine) in two cases (Mitchell et $a l ., 1989 \mathrm{~b}$ ) and codon 319 (histidine $\rightarrow$ tyrosine) in a case (Inana et al., 1989) were found among Japanese patients. Considering the diversity of ethnic origins, OAT mRNA and antigen phenotypes of GA patients, the more OAT mutants will be found.

The RP mainly affects the retina and choroid. It is suggested that a gene which causes chorioretinal disorders mainly expresses in the retina and choroid and plays important roles in retinal function. There are candidate genes; rod and cone visual pigments, $G$ proteins, cyclic GMP phosphodiesterases, interstitial retinol binding protein (IRBP), cellular retinol binding protein (CRBP) etc., for detecting light and triggering physiological visual signals that is processed and sent on to the brain. Some of the genes or cDNAs 
encoding these molecules have been cloned. With rigorous linkage studies and a host of candidate genes, the molecular defects leading to RP and other forms of RP are likely to be revealed in near future. Research Committee on Chorioretinal Degenerations, The Ministry of Health and Welfare of Japan, has also doing research since 1977 to study the causes and the possibility of therapy, and to establish clinical and etiological diagnosis including DNA of RP.

\section{SYMPOSIUM II. DNA Analysis}

SII-1. ORNITHINE TRANSCARBAMYLASE DEFICIENCY-PHENOTYFE TO GENOTYPE. Ichiro MATSUDA (Dept. Pediatr., Kumamoto Univ. Med. Sch., Kumamoto $860)$

The incidence of the five major disorders of the urea cycle was estimated to be 1 in 48,000 live births in Japan; The incidence of ornithine transcarbamylase (OTC) deficiency (X-linked inheritance) was the highest, the rate being 1 in 80,000 live births. We did a retrospective study of urea cycle disorders we encountered clinically between 1978-88. The 32 male patients found to have the disease were classified into two groups depending on age-at-onset; neonatal-onset group (0-28D) and late-onset group (>28D). In the first group, 2 patients with $0 \%$ of OTC had a nonsense $\mathrm{C} \rightarrow \mathrm{T}$ mutation in exon 5 , and one patient with $8 \%$ of OTC had a missense $A \rightarrow T$ mutation in exon 6 (Val-Asp). In the second group, 2 patients with $20 \%$ of OTC had a missense $\mathrm{C} \rightarrow \mathrm{T}$ mutation in exon 8 (Arg-Tryp). The relationship between genotypes and phenotypes in OTC deficiency is the subject of on going study.

SII-2. DNA ANALYSIS ON CHROMOSOME MICRODELETION SYNDROMES: APPROACH TO UNDERSTANDING THE CAUSES OF PRADER-WILLI (PWS), ANGELMAN (AS), AND LANGER-GIEDION (LGS) SYNDROMES. Norio NIIKAWA (Dept. Hum. Genet., Nagasaki Univ. Sch. Med., Nagasaki 852)

PWS, AS and LGS are considered as contiguous gene syndromes, since they are often associated with chromosome microdeletions and represent a variety of clinical manifestations. However, the causes of the syndromes remain unknown. PWS and AS show similar cytological deletions but the opposite parental origin of deletion, i.e., paternal origin in PWS and maternal origin in AS. In addition, uniparental disomy has been found in karyotypically normal PWS patients. Thus, the "genomic imprinting" hypothesis has been proposed that parental genes localized at $15 \mathrm{q} 11$-q12 are differently imprinted in epigenetic process and gene behaviors are different in offspring: a loss of the paternally-derived allele may lead to PWS, while a loss of the maternally-derived allele may result in AS. In this mndel it in ..... ned that the alternative phenotype of PWS would be AS.

IS and 13 AS patients molecular-genetically. DNA deletions were of PWS patients and in $8(61.5 \%)$ of AS patients, using 6 DNA 11-q12 as probes. Excess gene doses were observed in 3 PWS paon two PWS patients confirmed uniparental disomy. Deletion 